\title{
Aguas diversas. Derechos de agua y pluralidad legal en las comunidades andinas/
}

\author{
Diverse Waters. Water rights and legal pluralism \\ in Andean communities ${ }^{1}$
}

\author{
Rutgerd Boelens \\ Pontificia Universidad Católica del Perú \\ y Universidad de Wageningen, Países Bajos
}

Por el aumento generalizado de los conflictos por el agua, los derechos a este recurso se han vuelto ejes en los debates, reformas y programas de intervención hídrica. Los gobiernos, las agencias de desarrollo y los centros expertos tienden a considerar los 'derechos de agua' como meras cajas negras estándar que se yuxtaponen a los marcos de las ciencias técnicas y económicas positivistas. Pero mucho más allá de los manuales y las regulaciones de riego, hay otro mundo del agua íntimamente ligado a la vida diaria de gente real, hombres y mujeres que usan ese agua. Este artículo estudia cómo los colectivos de usuarios en la región andina practican una enorme variedad de derechos de agua y formas de gestión, como híbridos locales-nacionales-internacionales que se crean y afirman en los territorios hídricos locales, embebidos en contextos históricos y cultural-políticos.

Palabras Claves: Derechos de agua; Gestión de agua; Sistemas de riego; Pluralidad legal; Culturas hídricas; Identidad; Relaciones de poder; Comunidades campesinas; Andes.

Water rights and property relations have become pivotal issues in water debates, reforms and intervention programs. Governments, development agencies and expert centers tend to consider 'water rights' as merely standard black boxes that juxtapose the frameworks of positivist technical and economist water science. But far beyond universal manuals and irrigation regulations, there is another water world, entrenched in the everyday lives of real people, male and female water users. The paper makes clear how Andean user collectives practice an enormous variety of water rights and management forms, as localnational-international hybrids that are created and affirmed in local water territories, embedded in historical and cultural-political contexts.

KEYWORDS: water rights; water management; irrigation systems; legal pluralism; water cultures; identity; power relations; peasant communities; Andes

1 . Este artículo está basado en una revisión de secciones de The Rules of the Game and the Game of the Rules. Normalization and Resistance in Andean Water Control, Paises Bajos, 2008, Wageningen University. 


\section{Introducción: la interacción entre repertorios normativos}

Al aumentar la escasez de agua y los conflictos en muchas regiones del mundo, el tema de los derechos de agua y de las relaciones de propiedad se ha vuelto gravitante y estratégico en los debates políticos y legislativos y en los programas de desarrollo rural. Sin embargo, es común observar que los legisladores y las agencias de desarrollo típicamente consideran los 'derechos de agua' sólo como aquellas reglas y disposiciones que aparecen en las leyes y políticas oficiales. Para las comunidades indígenas y campesinas, un derecho de agua tiene también muchos otros componentes que son creados, reconfirmados y recreados en procesos históricos específicos a la localización, dentro de contextos políticos y culturales.

Los sistemas de riego gestionados por comunidades campesinas e indígenas en los Andes, objeto de este análisis, típicamente muestran una enorme diversidad de reglas, derechos y formas organizativas. Es una consecuencia intrínseca de la adecuación de la gestión y la infraestructura hidráulica a los requerimientos sociales y agrofísicos de cada localidad. Luego, son el resultado de las negociaciones entre los usuarios de agua y de los encuentros entre usuarios y agentes oficiales en cada sistema de riego, y de la confrontación con estructuras de poder locales y más amplias. De este modo, la interacción entre los diferentes marcos sociolegales es crucial. El derecho de agua en el riego campesino e indígena encarna combinaciones particulares de elementos de varias fuentes normativas y formas organizacionales (oficiales y no oficiales) que interactúan en el campo de cada sistema de riego específico. La mayoría de las veces, gran parte de estos derechos y reglas locales no están escritos aunque usualmente consisten en patrones claros y socializados de normas que son parte de la memoria colectiva y del marco de referencia locales.

Consecuentemente, para poder entender la gestión del riego en los Andes es crítico penetrar en estas raíces normativas plurales, dentro de su perspectiva histórica y cultural y de su actual constelación de poderes. Así, no es suficiente enfocar solamente sobre el nivel local comunitario o intercomunitario. El entender los repertorios sociolegales locales requiere entender cómo las normas oficiales del Estado y las leyes de los proyectos se han entreverado en las comunidades locales. Las leyes externas son una fuente poderosa de normalización en los sistemas de riego gestiona- 
dos localmente. ${ }^{2}$ Es usual ver que las instituciones estatales y sus funcionarios tienen poco conocimiento sobre las constelaciones de derechos de agua locales o simplemente las deniegan y se basan en sus propios antecedentes normativos. Además, ya que la ley estatal constituye una fuente de poder importante, las comunidades y los usuarios individuales del agua a menudo buscan estratégicamente usar sus reglas, derechos y procedimientos para su propia conveniencia.

La ley estatal, por tanto, constituye una amenaza y una oportunidad para las comunidades usuarias de agua en los Andes. Éstas la afrontan como un reto a su autonomía y como una herramienta para una eventual redistribución. A pesar de los recientes cambios constitucionales hacia un reconocimiento legal del multiculturalismo y de los derechos específicos a la localidad en los países andinos, el sistema normativo estatal se fundamenta en una aplicación general y uniformizante de la ley. Expresa la igualdad de todos los miembros de la sociedad en términos de derechos y obligaciones, generalmente sin excepción alguna que pudiera reconocer la validez de los diferentes subsistemas normativos. Particularmente las Leyes de Agua y sus Reglamentos tienden a seguir un modelo jurídicopolítico monista, que no reconoce los principios culturales locales de la gestión del agua ni concede validez a necesidades o formas organizativas particulares.

Pero la ideología de la ley oficial no se materializa en la práctica de manera simple a través de las formas que sus autores habían supuesto. La manera en que se ponen en vigor los derechos oficiales y su efectividad variará de acuerdo con la situación y el contexto. Esto se debe a la existente interacción mencionada en la cual, por un lado, funcionarios estatales responsables de la implementación legal interpretan los derechos oficiales y los distorsionan en la práctica, mientras que, por otro lado, los supuestos receptores son altamente heterogéneos en su aceptación de los derechos oficiales.

Luego hay una fuerte interacción entre los sistemas normativos locales ya existentes (por ejemplo, la ley estatal, leyes religiosas y culturales, y normas consuetudinarias) y los repertorios de derechos generados $\mathrm{o}$

2 La influencia de la ley estatal también difiere según el país: en términos generales, los sistemas de riego en Bolivia han sido históricamente menos afectados por la legislación estatal que en países más centralizados como el Perú, Ecuador y Chile. 
impuestos por los múltiples proyectos de riego, que a menudo implantan sus propios criterios para la gestión del riego. ${ }^{3}$

Como consecuencia de los procesos de interacción, normalización y resistencia, en la práctica del riego campesino e indígena en los Andes podemos encontrar normas y sistemas normativos que operan dentro de la ley, otros que lo hacen fuera de ella (sin apoyo legal; ni aceptados ni prohibidos por la ley) y otros que actúan contra la ley, con sus propias reglas que son ilegales respecto a la legislación oficial. ${ }^{4}$

En palabras de Moore, podemos analizar los marcos sociolegales de los sistemas de riego andinos como 'campos sociales semiautónomos'. Este campo "tiene capacidades de hacer reglas, y los medios para inducir o forzar el cumplimiento; pero está al mismo tiempo establecido en una matriz social mayor que puede afectarlo e invadirlo, y así lo hace". 5 Son semiautónomos no solamente porque pueden ser afectados por normas y fuerzas en efecto bajo otros sistemas normativos, tales como el marco legal nacional, sino también porque los usuarios de agua pueden movilizar estas normas externas, o amenazar con hacerlo, al enfrentarse con otros actores. Sin embargo, también tienen cierto grado de autonomía porque las normas legales o 'externas' tienen una función y un rango limitados dentro de la esfera normativa local, y sus propios derechos y obligaciones son con frecuencia fuertes y bastante importantes.

Especialmente cuando surgen conflictos, los interesados seleccionan estratégicamente de sus sistemas sociolegales las normas, reglas y procedimientos que puedan legitimar y reforzar sus reclamaciones particulares. ${ }^{6}$ Las posibilidades de los usuarios de agua de 'ir de compras' entre los diferentes sistemas de normas dependen tanto del poder que sean capaces de acumular para sostener y legitimar esas reclamaciones, como de la acepta-

3 Véanse Franz von Benda-Beckmann, Keebet von Benda-Beckmann y Joep Spiertz: "Equity and legal pluralism: taking customary law into account in natural resource policies", en Rutgerd Boelens y Gloria Dávila (eds.): Searching for equity, Assen, Van Gorcum, 1998, pp. 57-69; Rutgerd Boelens, David Getches y Armando Guevara (eds.): Agua y Derecho. Políticas hídricas, derechos consuetudinarios e identidades locales: Quito y Lima, Abya Yala-IEP, 2006; Jan Douwe van der Ploeg: El Futuro Robado. Tierra, Agua y Lucha Campesina: WALIR, Quito y Lima, Abya Yala-IEP, 2006.

4 Patricia Urteaga y Rutgerd Boelens (eds.): Derechos Colectivos y Politicas Hídricas en la Región Andina: Quito y Lima, Abya Yala-IEP, 2006; Alberto Wray: "El problema indígena y la reforma del Estado", Derecho, pueblos indígenas y reforma del Estado, Quito, Abya Yala, Colección Biblioteca Abya Yala, Vol. 2, 1993.

5 Sally F. Moore: "Law and social change: the semi-autonomous social field as an appropriate subject of study", Law and Society Review, Vol. 7, No. 4, 1973, p. 720.

6 Benda-Beckmann et al.: "Equity and legal pluralism...", p. 63 
ción general de las comunidades usuarias de agua de la legitimidad del sistema normativo 'externo' en cuestión.

Para entender la cuestión de los derechos de agua 'vivientes' como la médula de los sistemas autogestionados en los Andes, en este artículo se desarrolla un marco para analizar estos repertorios normativos como sistemas de complejidad organizada. Revela las múltiples capas del acceso y control del agua y cómo éstas se vinculan a fuentes y marcos sociolegales interactuantes. En las secciones siguientes se explica cómo estos repertorios ligan facultades, tareas, condiciones y reglas de operación y mecanismos para adquirir el agua; se relacionan con formas de legitimidad y autoridad; distinguen entre derechos de uso internos y externos y toman forma concreta en el proceso de traducción de derechos de referencia hacia 'derechos activados y materializados'. Se concluye que, además, los contenidos y la distribución de los derechos son, al mismo tiempo, objetos de contestación interna y externa, productos de las relaciones de poder y una relación de poder en sí mismos.

\section{Los derechos de agua insertados}

En las comunidades andinas las reglas, derechos y tareas adjuntas al agua están cercanamente vinculadas con una gran diversidad de fuentes y marcos sociolegales a escalas local, nacional, regional y hasta internacional. Por lo tanto, también están embebidos en una gran variedad de formas organizativas para regular y distribuir el agua. Y la complejidad del cuadro de los derechos de agua no se restringe solamente a las prácticas de gestión de agua y riego. La asignación y distribución están entreveradas dentro de instituciones y redes sociales y políticas y algunas de ellas tienen poca relación con la gestión del agua en sí misma. Esta calidad a menudo se olvida en los programas de desarrollo, así como en las propuestas de cambio de la legislación sobre el agua y en las políticas funcionales de los recursos hídricos.

En la comunidad indígena de Chumug San Francisco, en Licto, provincia de Chimborazo, Ecuador, los fuertes conflictos impidieron durante largo tiempo la acción colectiva en la gestión hidráulica. Como es común para la mayor parte de las comunidades andinas, los días de trabajo comunitario para construir y mantener los canales de riego estaban introducidos en el conjunto más amplio de sus actividades. Cada familia tenía que con- 
tribuir en la construcción colectiva de vías, canales, terrazas colectivas, la cancha de fútbol, la escuela, la iglesia, etc., para consolidar la condición de "buen comunero". El cumplimiento de las obligaciones de membresía de la comunidad implicaba la obtención de derechos que todos los miembros tienen, entre ellos los del agua. Tradicionalmente, la comunidad estaba constituida por miembros que eran católicos, pero la región fue invadida por clérigos evangélicos, ecuatorianos y estadounidenses, desde los 1970s. Para 1995 una pequeña mayoría de miembros de la comunidad eran evangélicos y en la Asamblea Comunitaria esta mayoría decidió que las futuras mingas de la comunidad deberían dedicarse a construir la iglesia evangélica, mientras que los católicos rehusaron hacerlo y subrayaron la necesidad de trabajar en algo que fuera una necesidad colectiva general: la construcción de los canales del sistema de riego. Ambos grupos se amenazaron mutuamente con retirar los derechos de agua al otro. Los evangélicos argumentaban que los católicos no cumplían con sus obligaciones de minga establecidas por la mayoría, y que consecuentemente debían perder su condición y sus derechos como buenos miembros de la comunidad, mientras que los católicos defendían que construir una iglesia evangélica no era una labor de interés colectivo y que, además, los evangélicos se negaban a cumplir sus obligaciones relacionadas con los derechos de agua. Tras varios años de conflictos, cuando la falta de acción colectiva amenazó con romper la supervivencia económica de las comunidades, los grupos nuevamente se unieron en sus esfuerzos de minga bajo el liderazgo de un presidente fuerte y 'de mente abierta'. La colaboración retomó su importancia crucial, particularmente en el campo de la construcción y mantenimiento de la infraestructura de riego, el sistema de agua potable y las vías.

En las comunidades peruanas encontramos los derechos de agua con la misma característica de estar encajados en la esfera religiosa y cultural, no sólo en las 'creencias andinas tradicionales', sino también, entre otros, en la confrontación entre católicos y evangélicos. Por ejemplo, en las comunidades de Mollepata, Cuzco, los evangélicos rehusaron colaborar en las faenas, el trabajo comunitario e intercomunitario para construir y mantener la infraestructura de riego. Era común que en estas faenas, hasta entonces manejadas por los católicos en la región, no sólo se incluyeran elementos del culto a las deidades andinas, como compartir bebidas y pequeñas ofrendas a la Pachamama (Madre Tierra) y los Apus (dioses de las montañas guardianes del agua) sino que también en las faenas, al igual 
que las mingas en el Ecuador, el alcohol tuviera un papel importante. Durante la faena se consumía una gran cantidad de chicha y licor de caña y al final del día no sólo la calidad de la comida sino la cantidad de la bebida era un importante ingrediente para juzgar el éxito de la faena. Los evangélicos explicaron su falta de participación diciendo que a ellos les estaba prohibido ingerir alcohol y que la eficiencia de las faenas de los católicos era baja por la ebriedad. Cuando los segundos reclamaron que entonces los evangélicos debían pagar la multa de ausentismo (comunitariamente acordada) para que la comunidad pudiera contratar peones en su lugar, los ausentes respondieron diciendo que no iban a dar un dinero que sólo se usaría para comprar más alcohol. La acción colectiva y, por tanto, la sostenibilidad del sistema de riego estaban en riesgo, así como las nociones existentes sobre derechos de agua, insertos en la racionalidad de la comunidad sobre construcción y mantenimiento colectivo a través de faenas o mingas.

De lo anterior se deduce que las cuestiones de cómo obtener derechos de agua, la definición de sus contenidos y su asignación hacia las familias usuarias — aparte de reflejar las circunstancias climáticas, agroproductivas y geofísicas - están fuertemente influenciadas por las normas sociales que regulan las prácticas de distribución y redistribución en otras esferas comunitarias (no de riego). Éstas tienen que ver con, por ejemplo, los derechos y obligaciones generales de la comunidad, las relaciones de familia y género, las estructuras de poder, las formas de organización históricamente generadas, y los ritos y creencias locales. ${ }^{7}$

Así, la formulación y puesta en vigor de los derechos de agua están cercanamente introducidas en los contextos locales y forman parte de los sistemas culturales históricamente establecidos con sus propios valores, significados y símbolos. También, en muchas comunidades, la identidad comunitaria está fuertemente vinculada con una historia compartida de luchas por los derechos de tierra y agua. Estas múltiples formas de inserción en contextos locales determinan la naturaleza y la función de los derechos de agua. También se relacionan fuertemente con la identidad de las

7 Xavier Albó: Iguales aunque Diferentes: La Paz, CIPCA, 2002; Paul H. Gelles: "Cultural politics and local resistance in highland irrigation development", en Rutgerd Boelens y P. Hoogendam (eds.): Water Rights and Empowerment, Assen, Van Gorcum, 2002, pp. 22-35; Milka Castro Lucic: "Local norms and competition for water in Aymara and Atacama communities, Northern Chile", en Ibidem, pp. 187-201; Ton Salman y Annelies Zoomers (eds.): Imaging the Andes: Shifting margins of a marginal world: Ámsterdam, Aksant, 2003. 
comunidades poseedoras de derechos de agua y sus territorios - algo que he dado en llamar la 'identidad hidráulica'.

Los contenidos de los derechos locales sólo tienen sentido dentro de un contexto local y pierden su relevancia en otros contextos, por ejemplo, a escala de los marcos legislativos nacionales. ${ }^{9}$ Es común ver que las políticas y los discursos (inter)nacionales buscan el decaimiento de los derechos de agua locales y minar la identidad hidráulica local, lo que a su vez es un proceso de des-identificación. Las comunidades, por su parte, luchan por impulsar sus derechos de agua y por defender y reconstruir su identidad hidráulica.

\section{Derechos colectivos e individuales}

Con arreglo al derechohabiente, los derechos de agua de riego en las comunidades andinas presentan la forma de derechos de agua colectivos o individuales. ${ }^{10}$ Respecto a esto prevalecen muchas definiciones en la literatura en las cuales los derechos individuales son a menudo confundidos con los derechos privados. Hemos definido los derechos de agua colectivos como las demandas de uso y control de ese líquido básico por parte de la organización de usuarios en un sistema de riego frente a otras partes (individuales o colectivas) cuyos intereses pueden chocar con los suyos propios. Estos derechos también determinan las formas y condiciones colectivas para acceder a la fuente de agua y a las prerrogativas y cargas asumidas por el grupo ante terceros. Los derechos individuales de agua, en cambio, están dentro de cada sistema, estableciendo relaciones por el acceso a ella entre los diferentes usuarios y sus respectivos derechos, privilegios y obligaciones. ${ }^{11}$

Así, particularmente los derechos individuales de agua establecen relaciones para el uso comunitario entre los y las regantes asociados a ese propósito, determinando los derechos y las obligaciones para cada uno. La necesidad de definir claramente los derechos y las obligaciones es una con-

8 Véanse Rutgerd Boelens y Bernita Doornbos: "The Battlefield of Water Rights. Rule Making Amidst Conflicting Normative Frameworks in the Ecuadorian Highlands", Human Organization, Vol. 60, No. 4, 2001, pp. 343-355; Rutgerd Boelens: "The politics of Disciplining Water Rights", Development and Change, Vol. 40, No. 2, 2009, pp. 307-331.

9 Franz von Benda-Beckmann et al.: "Equity and legal pluralism...".

10 Lily Beccar, Rutgerd Boelens y Paul Hoogendam: "Water rights and collective action in community irrigation", en Rutgerd Boelens y Paul Hoogendam (eds.): Water Rights ...., , pp. 1-21

11 Ibidem, pp. 3-4. 
secuencia directa del hecho de que el riego es, y sólo puede ser, visto como una actividad organizada colectivamente en la mayor parte de las regiones andinas. Es la conciencia de cada individuo que requiere de insumos de los demás para garantizar que el sistema trabaje y así se asegure su propio acceso al agua (una 'reciprocidad contractual colectiva'). Por ello, los intereses de cada uno y de todos los usuarios y usuarias comprenden una yuxtaposición de intereses aglomerados e interdependientes que, en caso óptimo, generan una red organizacional capaz de autogestionarse.

En los sistemas comunitarios andinos, quien tiene un derecho individual es la unidad familiar, que está generalmente, pero no siempre, representada por un miembro de la familia. Esto tiene obvias implicaciones en términos de las relaciones de género ya que no sólo la ley positiva sino también muchas formas locales y consuetudinarias de legislación en los Andes establecen que los representantes de la familia sean los hombres, excepto en familias lideradas por mujeres como, por ejemplo, en el caso de viudas, divorciadas o solteras que se encargan de su tierra regada.

Los derechos de agua colectivos, a su vez, a menudo tienen una (no menos importante) función externa fuera del sistema de riego. En este contexto, hablamos de los derechos colectivos de un grupo con respecto a otras partes que pudieran estar interesadas no sólo en usar el agua sino también en una serie de otros derechos relacionados con el uso del agua, tales como servidumbres de acueductos, derechos de vía para sus fuentes y derechos de toma de decisión sobre los recursos hídricos. Los terceros pueden ser usuarios individuales, otras colectividades regantes, otros sectores usuarios dentro de la cuenca hidrográfica o incluso grupos de no-usuarios con un interés particular en la creación de reglas relacionadas con el agua. Así, la función externa de los derechos colectivos está también reflejada con frecuencia en la defensa de los sistemas de derechos locales, su autoridad y su autonomía frente a otros sistemas normativos, tales como la legislación estatal y los regímenes de privatización de derechos orientados al mercado. ${ }^{12}$

A diferencia de los derechos entregados por el gobierno (o comprados en mercados de agua) que suelen estar relacionados con individuos,,$^{13}$ los derechos de agua en los sistemas comunitarios están generalmente entrega-

12 Ibidem

13 Particularmente las regulaciones oficiales suelen establecer que el derecho-habiente está definido como 'jefe de familia', lo que usualmente significa un hombre. Así la ley nacional introduce y refuerza las desigualdades de género y debilita las nociones de familia compartida y de propiedad común. 
dos a las familias al pertenecer a una colectividad y al cumplir con las correspondientes obligaciones comunales. En los Andes, así como en muchas partes del globo, las familias usuarias construyen su identidad al ser miembros cumplidos de la comunidad y de los sistemas de riego y, por lo tanto, los derechos de cada individuo se derivan y están insertados en los derechos y deberes colectivos. El derecho es normalmente intransferible por decisión individual porque pertenece a la colectividad; cuando alguien deja de usar agua, el derecho revierte a la comunidad. De manera similar, como hemos visto, las normas del uso de agua son parte del conjunto general de normas comunitarias, de modo que las infracciones en este área pueden ser también penalizadas en otras esferas de la vida comunitaria, y viceversa. ${ }^{14}$

En contraste, en aquellas regiones y sistemas donde los derechos de agua se han individualizado a lo largo del tiempo, los derechos y deberes son consecuentemente 'desinsertados' y se separan de otras esferas de la vida social comunitaria. Ser un regante en estos sistemas significa que se deben cumplir las obligaciones de riego específicas de cada uno, lo que no se puede intercambiar por contribuciones en otros ámbitos de la vida. El grado de poder que uno tiene sobre los propios derechos de agua también varía: en este caso, el derecho de uso de una porción dada del flujo o el volumen del riego con frecuencia también implica el derecho de transferencia: por préstamo, arrendamiento o incluso a veces por venta, ya sea bajo ciertas reglas de la organización local o no. A menudo coincide con la creciente intervención de actores con poder económico para comprar derechos de agua dentro del sistema. En Chile, por ejemplo, la individualización de esos derechos de agua está legislada, lo que significa que los usuarios compiten entre ellos dentro de un sistema de riego que solía ser gestionado colectivamente. ${ }^{15}$

\section{Mecanismos de adquisición de derechos}

A más de la cuestión de lo que consiste un derecho de agua en un sistema de riego dado, los colectivos de usuarios tienen que estar de acuerdo sobre cómo adquirir derechos de agua. Las diferentes autoridades y marcos

14 Beccar et al.: "Water rights and collective action...".

15 Ibidem. Véanse también Milka Castro Lucic: "Local norms and competition ...; Ingo Gentes: "La interacción de los derechos locales e indígenas de agua con la legislación chilena", en Rutgerd Boelens, D. Getches y A. Guevara (eds.): Agua y Derecho, Quito y Lima, IEP, 2006, pp. 255284; Jan Hendriks: "Water as private property. Notes on the case of Chile", en Rutgerd Boelens y G. Dávila (eds.): Searching for Equity, Assen, Van Gorcum, 1998, pp. 297-310. 
sociolegales que regulan los sistemas de riego en los Andes establecen y reconocen diferentes mecanismos para obtener derechos de agua. Cada forma de poner en vigor estos mecanismos depende de si los usuarios y sus comunidades reconocen o no la legitimidad de las autoridades que regulan estos mecanismos y si son capaces de defender su enfoque sobre los derechos en la práctica, internamente y frente a terceros. A menudo los derechos de riego están vinculados a los derechos de tierra y, por lo tanto, los cambios en los títulos de tierras (por ejemplo a través de la reforma de tierras) podrían alterar la distribución de los derechos de propiedad del agua. Pero, ya que los marcos de los derechos de agua son campos sociales relativamente autónomos, el vínculo con los derechos de tierras de ninguna manera es un hecho dado. Básicamente hemos encontrado y distinguido entre las siguientes categorías de mecanismos para acceder a los derechos de agua en los sistemas de riego andinos:

- Una concesión de derechos de agua: estos títulos de agua se entregan por parte de la administración estatal. La propiedad formal del recurso permanece en manos del Estado, pero los usuarios (colectivos o individuales) reciben el derecho de uso, distribución y gestión del agua de manera interna, por un periodo determinado. El estado usualmente cobra tarifas de concesión y, al ser el dueño definitivo, tiene el dominio final sobre el agua (según los acuerdos, podría retirar los derechos tras el periodo de concesión, incluir otros derechohabientes, transferir la propiedad de la infraestructura, etc.).

- Derechos históricos: son los que se refieren a reclamaciones que consideran una forma de 'apropiación previa'. Están legitimados sobre la base del reconocimiento de la adquisición por parte de los primeros usuarios en el pasado, que pueden ser colonos o pueblos originarios indígenas.

- Derechos socioterritoriales: son derechos sobre los recursos hídricos que se originan o fluyen a través de un territorio sociogeográfico (por ejemplo, los 'derechos de riberas' que se basan en la posesión de tierras con una fuente de agua o localizadas a lo largo de una corriente de agua). El ser habitantes de un socioterritorio al cual la fuente de agua 'pertenece', legitima las reclamaciones de títulos colectivos e individuales.

- Transferencia de derechos de agua de un derechohabiente a otro. De acuerdo con el marco normativo del cual partan los usuarios, habrá 
reglas muy específicas para establecer qué mecanismos de transferencia se consideran legítimos. Los mecanismos de transferencia pueden darse diversamente, por ejemplo, a través de compra-ventas, arrendamientos, trueques, donaciones, herencias o matrimonios, y cada mecanismo está vinculado a sus normas particulares (especialmente en cuanto a obligaciones y restricciones) sobre las condiciones de la transferencia, el universo de posibles beneficiarios de ella, etc. En general, las transferencias individuales están insertas dentro del régimen de propiedad colectiva que establece que los derechos no pueden ser 'sacados del sistema'. En muchas comunidades, vender y comprar derechos de agua está prohibido incluso dentro del sistema para evitar la acumulación de derechos de agua en pocas manos, así como la pérdida del control comunitario sobre la asignación del agua. La venta de la misma agua, por ejemplo de un turno de riego, generalmente encuentra menos restricciones.

- Adquisición de derechos de agua por la fuerza: la historia andina está repleta de incidentes de derechos de agua expropiados a comunidades indígenas y campesinas a través de fuerza coercitiva ejercida por los grupos poderosos (terratenientes, compañías mineras, empresas agrícolas, etc.).

- Inversión de los usuarios: las comunidades y las familias invierten sus propios recursos (por ejemplo en especie, mano de obra, capital, tiempo y contribuciones intelectuales y rituales) para construir o rehabilitar las instalaciones de riego, creando de esta manera derechos de agua.

Dentro de una región dada, es común encontrar varios de estos mecanismos diferentes trabajando simultáneamente. ${ }^{16}$ Es más, hay una interacción entre estos mecanismos; pueden reforzarse u oponerse unos a otros. Un ejemplo ampliamente distribuido en las regiones andinas se relaciona con la unión de fuerzas extraeconómicas y regulaciones estatales. Por ejemplo, en el Ecuador, tras la nacionalización de los derechos de agua en los años '70, el gobierno, por medio del mecanismo oficial de concesión estatal, podría haberse opuesto a este mecanismo de toma por la fuerza,

16 Aparte de estos mecanismos, para cada sistema y fuente específica de agua se debe llenar una serie muy diversa de condiciones específicas para ganar derechos. Por ejemplo, obtener derechos de agua puede estar condicionado a: tener títulos de tierras en el área de riego; pertenecer a un grupo social específico (por ejemplo grupos de clase, etnia, género, parentesco o comunidad); ser un buen comunero, ser regante de un cultivo específico, en una zona ecológica dada o en cierta zona geográfica, etc. 
devolviendo los derechos a través de concesiones de agua a los derechohabientes originales, que reclamaban sus derechos por medio del mecanismo de demandas socioterritoriales. En la práctica, sin embargo, ha sucedido lo inverso: el mecanismo de concesiones estatales ha reforzado el de la toma por la fuerza. Otro ejemplo común de confrontación es la que se plantea entre mecanismos: muchos sistemas de riego en regiones conquistadas o colonizadas fueron construidos por gente campesina o indígena. Según los mecanismos andinos de 'derechos creados por inversión de usuarios', ellos deberían haber obtenido derechos de agua. Pero, a pesar de sus reclamaciones, grandes porciones de estos sistemas benefician a importantes terratenientes, que usaron el mecanismo de toma por la fuerza para hacer que esta gente trabajara y así obtener derechos para sí mismos.

\section{Los contenidos de los derechos de agua}

\section{Derechos de acceso y control}

Como se detalló en la sección anterior, en la mayoría de los sistemas de riego andinos la asignación de agua está vinculada con la tierra y/o con las personas, pero a menudo se hacen arreglos específicos de acuerdo con los grupos de edad, las posiciones culturales-geográficas en la comunidad o los derechos previos. Los patrones reales de asignación han evolucionado históricamente con base en los principios locales de equidad y justicia, así como en las relaciones de poder y las contradicciones sociales.

Hemos definido los derechos de agua, en el contexto de la práctica de riego en sistemas andinos, como "demandas autorizadas para usar (parte d)el flujo de agua, incluyendo ciertos privilegios, restricciones, obligaciones y sanciones que acompañan a esta autorización, entre las cuales es clave el poder de participar en la toma de decisiones colectivas sobre la gestión y la dirección del sistema" ${ }^{17}$ Las condiciones, obligaciones, sanciones $\mathrm{y}$, de gran importancia, los privilegios de control y toma de decisiones vinculados con los derechos de agua, difieren según el sistema y el marco sociolegal.

Para entender conceptualmente el funcionamiento y el uso de los derechos de agua es esencial mirar los contenidos de los derechos de agua

17 Beccar et al.: "Water rights and collective action...", p. 3. 
cuando se manifiestan en situaciones particulares. Encontramos que los derechos de agua en sistemas controlados por los usuarios en la región andina usualmente establecen las siguientes cuestiones:

1) ¿quién tiene el derecho de usar el agua y la infraestructura hidráulica,

2) qué condiciones y criterios valen para obtener estos derechos,

3) cómo, cuándo, dónde, con qué propósito, en qué cantidad (y a veces en qué calidad) y con qué certeza le está permitido a cada usuario usar el agua,

4) cómo, cuándo y dónde les está permitido a los usuarios usar la infraestructura,

5) qué acceso tienen los derechohabientes a la información sobre la gestión del sistema,

6) qué obligaciones deben cumplirse para mantener los derechos,

7) qué sanciones siguen al desacato,

8) en qué grado pueden los usuarios tomar parte en las actividades organizativas, culturales y políticas relacionadas con la gestión del sistema,

9) quiénes son elegibles y cuáles son los criterios de elección para las posiciones relacionadas con la asignación del agua, la puesta en vigor de las reglas, la creación de propuestas de políticas y de regulaciones, y la gobernanza del sistema, incluyendo los procesos de negociación y de formación de alianzas con terceros, y

10) a qué grado cada usuario tendrá parte en la toma de decisiones colectivas acerca de los diversos temas relacionados con la gestión del sistema, particularmente la cuestión de la operación del sistema, aceptación de nuevos miembros y los cambios en las regulaciones internas y la futura propiedad del sistema? ${ }^{18}$

Ya que una definición precisa de estos aspectos es esencial, de modo que cada jugador sepa claramente con qué contribuir y qué recibirá en intercambio, ésta es una condición básica para una acción colectiva exitosa. La ausencia de criterios establecidos y aceptados como necesarios para el grupo, que pongan orden a la actividad de riego de la comunidad, llevará usualmente a una competencia descontrolada y a una falla en el trabajo de mantenimiento, lo que conducirá en el futuro a la destrucción del sistema de riego.

18 Boelens y Hoogendam: Water Rights and Empowerment. 
Los privilegios relacionados con los derechos de agua pueden ser agrupados, por similitud temática, en los siguientes subconjuntos:

1. Derechos de acceso y operación, que incluyen los privilegios de uso de parte del flujo, ${ }^{19}$ de uso de la infraestructura, de acceso a la información de gestión y de ocupación de posiciones para el desempeño de ciertas tareas de gestión. Son derechos de acceso y operación y se relacionan con el funcionamiento cotidiano (operación y mantenimiento).

2. Los derechos de toma de decisiones, que se refieren a los privilegios relacionados con tomar parte en las decisiones sobre la gestión del sistema, sobre la inclusión o exclusión de usuarios, sobre las modificaciones en el sistema hidráulico y sobre la transferencia de propiedad del agua o la infraestructura. ${ }^{20}$ Estos derechos se refieren a los permisos autorizados para controlar la gestión del agua de riego y decidir sobre el uso del recurso.

Dependerá de cada sistema qué tipos de derechos son parte de lo que sucintamente se denomina 'derechos de agua', y si es que los usuarios tienen derechos iguales o diferentes. Los derechos de agua se conceptúan en general como 'conjuntos de derechos' ya que el contenido de aquellos, aparte del privilegio de usar agua también se relacionan con varias capas de permisos, deberes, restricciones y sanciones, e involucran diversos niveles de autoridad y múltiples mecanismos para obtener los respectivos derechos. ${ }^{2 l}$

19 Aquí, los derechos de acceso se refieren a reclamaciones de retiro y uso de agua. Nótese que en otros usos de agua distintos del riego (como navegación o turismo) los derechos de acceso a veces son definidos como reclamaciones no extractivas para usar el agua. Además, los derechos de retiro y uso pueden ser tanto de consumo (por ejemplo consumo humano o para cultivos) como de no-consumo (por ejemplo, cuando se usan para (micro)generación de energía o para lavar o bañarse, después de lo cual el flujo de agua es devuelto al canal o río).

20 Beccar et al.: "Water rights and collective action...". Edella Schlager y Elinor Ostrom: "Property rights regimes and natural resources: A conceptual analysis", Land Economics, Vol. 68, 1992, pp. 249-262; Gerben Gerbrandy y Paul Hoogendam: Aguas y acequias, Cochabamba, Plural Editores, 1998.

21 Beccar et al.: "Water rights and collective action..."; véanse también: Benda-Beckmann et al.: Equity and legal pluralism..."; Dik Roth, Rutgerd Boelens y Margreet Zwarteveen (eds.): Liquid Relations. Contested Water Rights and Legal Complexity, New Brunswick, New Jersey y London, Rutgers University Press, 2005. 


\section{Obligaciones y sanciones}

En cuanto a las obligaciones de contraparte al goce de esos derechos, éstas implican en términos generales el cumplimiento de las normas del sistema en cuanto a uso y operación, contribuciones pecuniarias (tarifas, cuotas), mano de obra en la construcción y mantenimiento de la infraestructura hidráulica (mingas, faenas), contribuciones materiales (productos agrícolas, material de construcción), así como contribuciones intelectuales y organizadoras para sostener la gestión del sistema (asambleas, cargos, etc.). En muchos sistemas andinos las obligaciones abarcan no sólo el mundo de los seres humanos, sino también el de las deidades y la naturaleza sagrada, en términos de ritos asociados con el agua y su gestión. Éstos reafirman los derechos y relaciones de índole mutua, y crean una sensación de protección contra el castigo sobrenatural. Los ritos en la gestión del riego tienen con frecuencia una función de mantenimiento y están relacionados con la recreación de los derechos de agua.

La inserción de los derechos de agua en las estructuras sociales significa que en la mayoría de los sistemas autogestionados, los usuarios tienen también que ser miembros activos y tomar parte en tareas comunitarias fuera del ámbito de la gestión del agua.

Es usual que una obligación importante para poder acceder a los derechos de agua sea la de desempeñar un cargo en la gestión del agua en la comunidad. Los nombres en español, quechua y aymará difieren según el sistema y en varios de ellos se designan diversos cargos como, por ejemplo, los siguientes: yaku alcalde (alcalde o juez de aguas), relojero (el que cuida los tiempos), vocal (voceador o comunicador), jareador, unu kamayoq, rondador, aguatero o aguador (el que distribuye el agua), vigilante o tiyaq (guardián de los canales y el agua), etc. En muchas regiones andinas, según las prescripciones legislativas nacionales, los nombres se han formalizado, por ejemplo, bajo etiquetas tipo presidente, vicepresidente, tesorero, secretario, etc. No obstante, los contenidos difieren notablemente y, en muchos casos, los puestos tradicionales son los que tienen funcionalidad.

De acuerdo con el puesto, puede ser un servicio de una sola vez en la vida, a veces pesado pero al mismo tiempo honorable, o puede ser un puesto para el cual se puede ser elegido varias veces por la asamblea. A menudo los cargos rotan a través de los años en la mayor cantidad de miembros como sea posible, para compartir equitativamente los honores y las cargas, para evitar la concentración de conocimiento y poder de toma de decisio- 
nes en pocas manos y, más que nada, para mejorar la sustentabilidad del sistema al construir capacidades de liderazgo apropiadas entre un número grande de usuarios. Por supuesto que esto también depende fuertemente de las estructuras de poder prevalecientes en el sistema. Por ejemplo, en Cotahuasi (Perú), es común que los mayoristas (grandes terra- y aguatenientes) no contribuyan al mantenimiento por medio de su propio trabajo en las faenas, sino que paguen una cantidad desproporcionadamente pequeña de dinero en efectivo. Los minoristas proveen de la mano de obra. Además, la ambigua condición de los cargos de agua (pesados, honrosos y a veces poderosos) hace que en muchos casos haya manipulaciones y fuertes debates comunitarios y que no siempre sea la justicia social la que prepondere. Es más, a pesar de que los roles de género están cambiando rápidamente en los sistema andinos de riego, tradicionalmente la mayoría de los cargos ha estado destinada a los usuarios varones.

Con respecto a las tarifas que se cobran en los sistemas de gestión colectiva, éstas usualmente se mantienen a un mínimo y son impuestas para cubrir sólo aquellos gastos que están necesariamente relacionados con el mercado, como por ejemplo pagar ciertas reparaciones y materiales de construcción, el transporte y los viáticos cuando los líderes viajan a negociar con donantes u oficiales gubernamentales, o contratos de ingeniería hidráulica en un diseño complejo. Hasta cuando se trata de sobornos recolectados entre los usuarios para 'lubricar' las negociaciones o 'convencer' a los ingenieros oficiales o a otros actores externos, éstos a menudo consisten en bienes no comerciales, tales como productos y animales locales. Eso quiere decir que la mayoría de sistemas comunitarios en los Andes no construyen un fondo sustancial para cubrir las reparaciones más caras, especialmente en casos de calamidad. Esto se ha explicado con frecuencia como "la carencia de capacidad de planificar" de las comunidades andinas. Pero para controlar situaciones emergentes, la racionalidad del riego local se basa comúnmente en la movilización rápida y masiva de mano de obra y medios locales colectivos, no en un sistema de seguridad monetizada.

En principio, cuando alguien falla en el cumplimiento de las tareas asociadas con los derechos de agua, se aplican las sanciones, que pueden incluir la exclusión del turno de agua una vez o varias, el pago de multas, la demanda de contribuciones complementarias o la negación del derecho de agua por una o varias estaciones. Usualmente las sanciones son progresivas con el objeto de poner una presión creciente sobre los contraventores y también para darles la oportunidad de cambiar su comportamiento tras la infracción. 
Al contrario de los derechos de agua incluidos en los sistemas comunitarios, las regulaciones de riego estatales suelen ser uniformes, altamente funcionales y orientadas al pago monetario, es decir, están 'embebidas' en una racionalidad completamente distinta. El conjunto de derechos de los usuarios es mucho más restringido y sesgado hacia las obligaciones y los privilegios establecidos de arriba hacia abajo.

\section{Principios internos de asignación y modos de establecer padrones de turnos}

Aparte de la cuestión de cómo obtener derechos de agua, es decir, los mecanismos de adquisición, una cuestión importante es, lógicamente, cuándo (el tiempo) y cuánta agua será accedida por el derechohabiente (en términos de tasa de flujo, volumen, porción u otras expresiones), y qué tipo de principios rigen esta decisión. Durante muchos años de contacto con el campo encontré que la diáfana distribución mediante tres pasos de la provisión del servicio hídrico (asignación, planificación de turnos y distribución) es posible hacerla en los manuales de riego y las propuestas de proyectos, pero en aquellos sistemas gestionados por comunidades indígenas y campesinas, la distinción es menos clara: las definiciones y manifestaciones locales de asignación y establecimiento de los padrones y turnos de riego están comúnmente entreveradas. Por ejemplo, la idea de que 'las familias reciben agua de acuerdo con la mano de obra invertida en la construcción de la infraestructura de riego' (una regla con decenas de variaciones), apunta hacia que los asuntos de quiénes serán los derechohabientes (mecanismo de asignación) y cuánta agua deberán recibir. Otro principio, que establece que los derechohabientes reciben el agua en proporción con el área regable que poseen pero hasta un límite preestablecido (derechos de agua con "límite superior"), sirve para compartir la escasez entre todos: los pequeños riegan toda su tierra, los grandes sólo una parte hasta que llegan a ese techo. Otros principios distribuyen los turnos según prioridades sociales o políticas (por ejemplo, prioridad para los ancianos, o prioridad de acceso al agua de acuerdo con clase, género o casta), o según prioridades agrícolas y de seguridad social (prioridad a ciertos cultivos que se siembran en la zona regada).

Así, hemos encontrado más de quince principios fundamentales de asignación, cada uno muy distinto del otro, que son usados en las comunidades andinas. Por ello, a más de una forma totalmente diferente de pensar 
acerca de cómo adquirir derechos de agua y de los contenidos de los conjuntos de derechos en sistemas gestionados respectivamente por el estado o las comunidades, también las concepciones y procedimientos de planificación para hacer operativos estos derechos siguen una racionalidad diferente.

Tales principios son los ladrillos de complejos sistemas sociales. Son las reglas que establecen los términos básicos, las prioridades y/o las normas operativas en relación con el acceso de los derechohabientes a los flujos de agua. En un sistema de riego andino usualmente uno o algunos de estos principios forma el marco nuclear, pero hay muchas reglas adicionales que generan distintas matrices de combinaciones entre los principios básicos. El análisis y desenredo de los conjuntos de derechos en contextos particulares muestra que lo que parece ser un desorden incomprensible, inestable o irracional, inapropiado para la tarea de gobernar el ámbito altamente conflictivo del control del agua, en la práctica puede ser caracterizado como una complejidad organizada.

Para ilustrar brevemente algunas combinaciones posibles, de entre muchas, veamos la combinación 'las familias reciben derechos de acceso al agua según la mano de obra invertida en la construcción del sistema':

En el departamento boliviano de Oruro varios ayllus se juntaron en un programa de desarrollo de riego pequeño que se basaba en el diseño y estrategias de construcción interactivas, con base en las concepciones de derechos locales. ${ }^{22}$ Las comunidades decidieron sobre los asuntos de inclusión y exclusión de usuarios y sobre la tasa de inversión/beneficio, como es común en los sistemas comunitarios locales. Los principios para concretar los derechos de acceso al agua, sin embargo, eran bastante diversos a pesar de los antecedentes relativamente similares de las comunidades. En Jalaqueri todas las familias contribuían con idéntica cantidad de mano de obra y todos obtenían el mismo acceso al agua. En Yanuma, el ayllu decidió que el insumo en mano de obra por familia debía estar de acuerdo con el tamaño de la tierra de cada usuario y los derechos de agua iban de acuerdo con esta tenencia de la tierra. En Yucasa, el ayllu decidió que las familias tenían que contribuir con la misma cantidad de días de trabajo de construcción, a pesar de que la tenencia de la tierra no era igual y los derechos de agua se asignaron de acuerdo con esta tenencia diversa. En el ayllu de Lucumpaya-Pahua, como en Jalaqueri, la inversión en mano de obra de los usuarios era igual

22 Washington Claure, Zulema Gutiérrez y Paul Hoogendam: "Design and water rights. Small scale irrigation experiences in Oruro, Bolivia", en Boelens y Hoogendam (eds.): Water Rights..., pp. 173-186. 
para todos y los derechos de agua correspondientes también eran los mismos para cada usuario. Pero en este caso, a diferencia de los otros, los usuarios sin el estatus de miembro de la comunidad podían unirse para llenar el número de días de trabajo necesarios para construir el sistema, en vista de que la comunidad no tenía suficiente mano de obra propia. En Chillcani, a los nuevos usuarios se les permitía unirse y ganar iguales derechos de agua, pero sólo después de haber contribuido una cantidad extra de días de trabajo sobre los requeridos para cada usuario. A los usuarios de la cola del mismo sistema se les permitía contribuir sólo la mitad de los días de trabajo, ya que sus derechos de acceso al agua eran menos seguros.

Muchos de los principios y modos se refieren a características relacionadas con familias derechohabientes, y la asignación también se hace a derechohabientes concretos, incluso en aquellos casos en que las características de sus campos son decisivas. En contraste, los proyectos diseñados desde el Estado y las agencias de desarrollo usualmente asignan y distribuyen agua a la tierra regada o sólo a las áreas regables . A menudo el balance técnico entre las características agrícolas y físicas del sistema se sobrepone al balance social entre los usuarios humanos. Aquí tenemos uno de los temas centrales alrededor del cual difieren las percepciones de equidad de campesinos e ingenieros. Tales principios uniformes, entre otros estándares, son fundamentales no sólo dentro de los marcos burocráticos de los derechos, sino también para los enfoques basados en el mercado, particularmente el poderoso programa de políticas hídricas neoliberales en los países andinos. $^{23}$

\section{Derechos de uso doméstico y de uso externo}

Antes de implementar el recientemente construido sistema de riego de Licto, Ecuador, que había sido un sistema gestionado desde el Estado, la agencia nacional de riego envió a la organización de usuarios sus regulacio-

23 Eric Swyngedouw: "Globalisation or "glocalisation"? Networks, territories and rescaling", Cambridge Review of International Affairs, Vol. 17, No. 1, 2004, pp. 25-48; José Esteban Castro: Water, Power, and Citizenship. Social Struggle in the Basin of Mexico, Basingstoke y New York, Palgrave Macmillan, Houndmills, 2006; Hugo de Vos, Rutgerd Boelens y Rocío Bustamante: "Formal Law and Local Water Control in the Andean Region: A Fiercely Contested Field", International Journal of Water Resources Development, Vol. 22, No. 1, 2006, pp. 37-48; Karen Bakker: Beyond Privatization: Water, Governance, Citizenship, Ithaca, Cornell University Press, 2009. 
nes estándares que detallaban las recetas nacionales para la gestión del agua en los sistemas futuros: reglas, derechos, obligaciones y estructuras de autoridad. La junta intercomunitaria fue informada de que tenía que aprobar estas regulaciones. Pero, sabiendo que la firma del documento significaría una limitación de la autonomía y una negación de su propio conjunto de normas de gestión, la organización de usuarios entró en un largo proceso de análisis y discusión. Con apoyo de la ONG CESA, primero tradujeron la jerigonza ingenieril y luego cambiaron la regulación hacia un documento que no era perjudicial para sus autoestablecidas reglas, derechos y autoridades. Esto se presentó al gobierno como la regulación formal de los regantes de Licto. Mientras tanto, continuaron redactando su propia regulación interna, un documento no oficial pero que se convertiría en el verdadero marco de referencia para sus derechos y tareas relacionados con el agua.

Este caso ejemplifica el proceso que se da en la mayor parte de los sistemas en los Andes, cada vez que hay que formalizar o registrar reglas y derechos de operación propios, por ejemplo para legalizar la organización de usuarios de agua, para obtener créditos o concesiones de derechos de agua, o para ingresar en programas de desarrollo. Para entender los contenidos de los derechos de agua campesinos e indígenas es necesario, por lo tanto, distinguir entre derechos con propósitos de identificación externa y derechos con propósitos de regulación interna. El primer conjunto establece las reglas y derechos de un grupo de usuarios frente a grupos y agencias 'de afuera' y el segundo conjunto contiene las reglas y derechos que usualmente son mucho más precisos y establecen el marco interno que los usuarios requieren obedecer, de acuerdo con las autoridades locales y los acuerdos colectivos. Los derechos de identificación externa pueden consistir en conjuntos que siguen directamente las recetas nacionales pero, como el caso muestra, pueden ser elaborados localmente 'para consumo externo'. En tal caso, los derechos a menudo son definidos de manera estratégica, incorporando muchas veces elementos de las reglas oficiales con 'importancia estratégica'. Esto no implica que estas reglas sean practicadas también internamente.

\section{Derechos de agua y regímenes de propiedad}

Dependiendo de la organización social y política de los derechos de propiedad de agua (quién autoriza el derecho, qué regulaciones y poderes 
tiene la entidad autorizadora, qué reclamos y poderes están asociados con el derecho del usuario) podemos distinguir entre: regímenes de propiedad pública, regímenes de propiedad privada, regímenes de propiedad común y situaciones de acceso libre. ${ }^{24}$

En un extremo del espectro están los sistemas gestionados burocráticamente, en los cuales el Estado o una de sus agencias es el habiente de todos los derechos de toma de decisiones. El Estado determina así la asignación del agua, regula el uso del agua y decide sobre las modificaciones al sistema, inclusión y exclusión de miembros nuevos y la enajenación de la propiedad de la infraestructura y el agua. Al entregar concesiones de agua a individuos o grupos de usuarios, la propiedad permanece formalmente en manos estatales y los derechos de los usuarios se refieren usualmente a privilegios concernientes al acceso al agua y derechos operativos, no de decisión.

En el otro extremo del espectro encontramos los sistemas de propiedad privada. Todos los derechos de acceso y control están investidos en el propietario individual. Excepto en los casos de los dueños de bombas de pozos y de río, la gestión del agua basada en la propiedad privada no es muy común en las comunidades andinas de pequeños propietarios en vista de que el uso del agua de riego normalmente necesita de acción colectiva dentro de una forma particular de organización social. Sin embargo, tradicionalmente las haciendas han constituido instituciones de propiedad privada del agua basadas en la organización del trabajo forzado. Y en la actualidad el número de compañías privadas grandes que tienen concesiones de derechos de propiedad privada está creciendo rápidamente. En Chile estos derechos privados están legalizados en el sentido de propiedad real, ya que el derechohabiente tiene todos los derechos de uso, gestión y regulación del agua, de inclusión y exclusión de otros y de enajenación de estos derechos de propiedad. ${ }^{25}$

Un tercer tipo, los sistemas de riego de régimen de propiedad común, está íntegramente gestionado y mantenido por el grupo de usuarios. Éste es el caso de la mayoría de los sistemas de pequeña escala en los Andes. Son normalmente de propiedad colectiva de comunidades campesinas e indígenas, ayllus indígenas y cooperativas agrícolas que tienen control local sobre

24 Bryan Bruns, Ruth Meinzen-Dick y Claudia Ringler (eds.): Water Rights Reform, Washington D.C., International Food Policy Research Institute (IFPRI), 2005.

25 Castro: "Local norms and competition for water..."; Gentes: "La interacción de los derechos locales...". 
el rango completo de derechos de acceso, operación y toma de decisiones. De manera general, el aspecto de la gestión necesariamente colectiva es una característica importante que distingue el agua de muchos otros recursos que pueden ser de control individual.

Formalmente, las políticas hídricas suelen establecer un régimen de propiedad pública del agua a escala nacional (el agua es un recurso público) mediante el cual el agua a escalas inferiores puede ser mantenida en condiciones de derechos de propiedad pública, privada o común, o una mezcla de ellos. Así, es común que los regímenes de propiedad del agua estén parcialmente condicionados por las regulaciones del dominio público que restringen el poder de toma de decisiones privado o colectivo. Las orientaciones legales difieren fuertemente según el país. Las estructuras de propiedad reales, sin embargo, suelen desviarse notablemente de las estructuras formales. En la práctica, la mayor parte de los derechos de agua en los países andinos (con orientaciones de propiedad ya sea pública, privada o común) se acumulan en poderosos propietarios individuales, tales como terratenientes o empresas, que fundamentalmente tratan estos recursos como propiedad privada.

El debate académico sobre regímenes de propiedad se ha vuelto fuertemente sesgado y esencializado, especialmente desde que Hardin lanzara su bien conocido ataque en la "Tragedia de los Ejidos", ${ }^{26}$ en el cual, de manera errónea, analizó la tenencia de derechos colectivos como una especie de régimen de propiedad de acceso abierto para todo el mundo. Según la línea de pensamiento de Hardin, los nuevos hacedores de políticas se proponen contraatacar la 'tragedia' resultante de la sobreextracción y degradación de los recursos instalando un régimen basado en el control estricto de la propiedad pública sobre el agua, o un régimen de propiedad basado en derechos claros de propiedad privada gobernados por el mercado. El error principal está en considerar la gestión colectiva de los recursos naturales como un acceso abierto al recurso. Por el contrario, está gobernada por reglas y derechos estrictos de acceso y control colectivos. Pero, como respuesta al cuadro generalizador a la crítica esencializante de Hardin y sus seguidores, quienes abogan por la propiedad común a menudo han caído en el error opuesto e igualmente estereotípico: romantizar y reificar la existencia de regímenes de propiedad común.

26 Garrett Hardin: "The Tragedy of the Commons", Science, Vol. 162, 1968, pp. 1243-1248. 
La esencialización de los regímenes de propiedad empíricamente existentes puede que clarifique el debate teórico, pero ciertamente no ayuda a entender la práctica de los derechos hídricos en los Andes. Lo más común es la existencia de una articulación y una interacción dinámicas, en el sentido legal pluralista, de derechos que se originan en diferentes fuentes, marcos normativos o regímenes de propiedad. Esta interacción difiere por sistema de gestión de agua. Por ejemplo, los sistemas de riego de tamaño medio o grande en los Andes son usualmente co-gestionados y se caracterizan por alguna combinación de regímenes de propiedad público y común. ${ }^{27} \mathrm{El}$ tema de la confrontación, interacción y articulación de los diversos regímenes de propiedad se vuelve especialmente importante ahora que los debates actuales sobre política hídrica parecen coincidir en que las soluciones a la crisis del agua no deberían restringirse a los sistemas individuales sino que hay que encontrarlas a escala de usos y usuarios múltiples al nivel de la gestión de cuencas hidrográficas. Aquí se junta una serie diversa de marcos y regímenes de propiedad interactuantes y en conflicto.

\section{Condiciones fluidas y relaciones dinámicas: los derechos de agua en acción}

\section{Las dinámicas de los derechos de agua ${ }^{28}$}

A más de las impresionantes variaciones locales en los Andes, también la dimensión tiempo es un factor importante en la variedad de los derechos de agua que son modificados, histórica y actualmente, de acuerdo con los cambios sociales, económicos, ecológicos y hasta climáticos. La causa más ampliamente distribuida de los cambios graduales es el cambio demográfico, que trae consigo presiones internas sobre el uso del agua, concluyendo en algún momento en una redefinición de los derechos entre los usuarios.

27 Al discutir los regímenes de propiedad es de importancia gravitante considerar los niveles de gestión de agua a los cuales corresponden los derechos de acceso, operación y control: si los usuarios en sistemas autogestionados poseen derechos de operación y control concernientes a todos los niveles del sistema, en los sistemas gestionados por la agencia o cogestionados, sus derechos operativos pueden estar restringidos sólo a los canales secundarios o a los bloques terciarios. En los esquemas de tenencia burocrática pueden tener acceso sólo a derechos de operación a nivel del lote regado.

28 Sección basada en Beccar et al.: "Water rights and collective action...". 
Al igual que el incremento de usuarios, un decremento es también la causa de cambios mayores en los sistemas de riego y derechos de agua. La migración permanente o temporal es un fenómeno fundamental en muchas regiones andinas. La migración a las ciudades u otros países especialmente de los varones, que generalmente son los derechohabientes formales y representantes de la familia en la organización de usuarios, implica un cambio estructural en la gestión de muchos sistemas comunitarios. La ausencia de hombres, a veces seguida de la ausencia de muchas mujeres también, genera nuevos desafíos en la distribución de los derechos de uso de agua, en la resolución de escasez de mano de obra para operación y mantenimiento, y en la redefinición de los roles y tareas de riego y organización.

Otra causa gradual de cambio es la reorientación de la producción, que entraña una nueva distribución de las cantidades y las frecuencias de agua de acuerdo con los nuevos requerimientos. Cuando los sistemas, por razones de mercado, están diversificando su producción o, en contraste, cuando cambian desde una producción diversificada para autoconsumo hacia un monocultivo para el mercado, requieren de otras frecuencias de riego y volúmenes de agua y, por lo tanto, de una redefinición de por lo menos los contenidos de los derechos de agua referidos a los padrones de distribución. El mismo proceso se observa también como resultado del cambio climático en los Andes, que genera mayor escasez de agua (a la par que grandes inundaciones).

Las causas de cambio abrupto incluyen, por ejemplo, los procesos de reforma agraria, en los que la nueva distribución de tierras también incluye cambios en la distribución del agua. En muchos de estos procesos, los dependientes de las antiguas haciendas se ven forzados a encontrar maneras de distribuir el agua entre ellos mismos, usualmente sobre la base del patrón de distribución aplicado durante el sistema hacendario, adoptando sus percepciones de justicia. En otros, el proceso de redistribución de agua incluye grupos que no eran parte del régimen hacendario y que ahora demandan el regreso del agua usada por sus ancestros y el reestablecimiento de su sistema normativo propio. ${ }^{29}$

Los cambios en la legislación y la política hídricas, tales como la privatización o la transferencia de la gestión del sistema de riego desde el

29 Rocío Bustamante: "Pluri-, multi-issues in the reform process: towards new water legislation in Bolivia", en Water and Indigenous Peoples, Paris, WALIR, UNESCO, 2006, pp. 126-143; María Teresa Oré: Agua, bien común y usos privados. Lima, PUCP, 2005. 
Estado a los usuarios, son otros factores de transformación en muchos sistemas de riego en los Andes. Los vacíos en poder y capacidad organizadora, así como la carencia de derechos claros y bien publicitados en la nueva situación, han llevado a menudo a importantes dificultades en la gestión de estos sistemas. De modo similar, en algunas regiones la nacionalización de la propiedad del agua hace varias décadas, y luego la privatización en el presente, han causado un grave impacto sobre los derechos de agua. ${ }^{30}$

Estos procesos de cambio generan discusiones y negociaciones entre los usuarios y usuarias acerca de cómo adaptar sus normas a las nuevas circunstancias. El hecho de que muchos sistemas de riego en los Andes tengan una larga historia de operación muestra que, en medio de todos estos cambios, han sido capaces de mantener una sustentabilidad dinámica. Un ingrediente clave para esto es la capacidad organizativa para adaptarse mediante arreglos adecuados que permitan continuar con la operación del sistema. Esta sustentabilidad, sin embargo, no quiere decir que no haya disputas en estos sistemas relacionados con la igualdad en la distribución del agua. El tema principal para la sustentabilidad es tener un consenso básico entre los usuarios acerca de la legitimidad de los derechos de agua y la capacidad para ajustarlos. Otro ingrediente principal es el hecho de que tanto el proceso de toma de decisiones sobre los derechos como la distribución misma deben corresponder substancialmente con lo que se define localmente como equitativo.

\section{La naturaleza fluida del agua y la apariencia versátil de los derechos de agua}

Las dinámicas de los derechos de agua también salen al frente en el funcionamiento cotidiano de los sistemas de riego. Aquí el tema de los

30 Véanse Bakker: Beyond Privatization...; Castro: "Local norms and competition for water..."; Leontien Cremers, Marjolein Ooijevaar y Rutgerd Boelens: "Institutional reform in the Andean irrigation sector: Enabling policies for strengthening local rights and water management", Natural Resources Forum, Vol. 29, 2005, pp. 37-50; Jorge Armando G. Guevara: "Official water law versus indigenous and peasant rights in Peru" en Water and Indigenous Peoples, Paris, WALIR/UNESCO, 2006, pp. 126-143; Hendriks: "Water as private property..."; Tom Perreault: "Custom and contradiction: rural water governance and the politics of usos y costumbres in Bolivia"s irrigator movement”, Annals of the Association of American Geographers, Vol. 98, No. 4, 2008, pp. 834-854. 
'derechos de agua en acción' es esencial. El entendimiento de los conjuntos de derechos oficiales y localmente particulares, como he desarrollado en las secciones precedentes, es útil tanto para propósitos analíticos como por razones prácticas, porque sirve como marco referencial para derechohabientes y reclamantes, pero no es suficiente. Lo que pasa en la práctica real no puede ser fácilmente 'leído' en estos derechos. La asignación oficial de los derechos es a menudo diferente de los turnos y horarios de agua operacionales planeados por aquellos que están a cargo de la operación y mantenimiento del sistema. De la misma manera, la distribución real del agua difiere de estos horarios operacionales.

Así, la distribución del agua a los campos de cultivo difiere significativamente de los horarios planeados y de los patrones de asignación. Es en parte una consecuencia directa de las particularidades del agua como recurso. A diferencia de la tierra, casi toda el agua existe en un estado transitorio. La mayoría de las corrientes superficiales también son típicamente variables en el tiempo y en el espacio. Esto hace que no sólo cambie la disponibilidad del recurso con el paso del tiempo, sino que también fluctúen necesariamente las reglas y derechos vinculados a él. En periodos de escasez de agua los requerimientos de agua de riego son altos y en la mayoría de sistemas manejados comunitariamente las reglas son muy estrictas; pero en la estación de lluvia es común que los mismos usuarios interactúen sobre la base de reglas mucho más débiles y que incluso los afuereños tengan permiso de tomar el agua.

El uso del agua implica otra dificultad de controlarla efectivamente. La cantidad de agua disponible para un usuario es por tanto no sólo una función de los parámetros físicos, sino que principalmente depende de las prácticas de uso del agua de los otros usuarios. Incluso cuando los usuarios no tienen títulos legales o si es que no han cumplido con todas las obligaciones, todavía pueden ser físicamente capaces de hacer que el agua se desvíe desde los canales hacia sus campos.

Dadas estas características variables y fluidas del recurso agua, hay mucho espacio para que los usuarios actúen de maneras que se separan de los derechos de agua legal y localmente establecidos. La distribución de agua, tal vez más que cualquier otro recurso, es típicamente objeto de regateo, negociación y disputa. Por lo tanto, para entender el control de agua no es suficiente ver la terminología formal, ya sea oficial o local, que define la condición de los derechohabientes. Tal entendimiento requiere de una visión de las prácticas reales de uso y distribución del agua. 


\section{La activación de los derechos de agua: derechos de referencia, derechos en acción y derechos materializados ${ }^{31}$}

Para entender la dinámica del derecho de agua en la práctica es necesaria una distinción analítica entre los diferentes grados de operativización del derecho de agua. Una persona o grupo de personas puede tener un derecho, pero esto no significa que esa persona automáticamente pueda en la práctica tomar ventaja de ello como se preveía. Sobre todo, los grupos con menor poder a menudo tienen dificultades no solamente en obtener derechos de agua, sino también en materializarlos. Por ello, en el análisis de las dinámicas de la gestión hídrica hemos distinguido entre las siguientes categorías de derechos: derechos de referencia, derechos activados y derechos materializados. Esta diferenciación conceptual es necesaria para capturar la diferencia entre los derechos oficiales (legales) o institucionalizados localmente, por un lado, y por el otro, los derechos reales que gobiernan la distribución concreta del agua y las relaciones sociales en las comunidades usuarias del agua. Así, es más apropiada que otras nociones más vagas como las distinciones entre lo formal y lo informal, o entre derechos de facto y de jure.

Los derechos de referencia son formulados de acuerdo con las normas y los principios prevalecientes en un marco normativo particular. Pueden ser generales (por ejemplo, las normas prescritas en las regulaciones hídricas nacionales) o específicas del lugar (por ejemplo, normas formalizadas relacionadas con un marco sociolegal local dado). Los derechos de referencia especifican el tipo de poderes que un derechohabiente tiene en términos de operación/acceso y de elementos de control, y también específica las características de los derechohabientes, por ejemplo, ser dueños de tierras, hombres o jefes de familia. En los Andes los derechos de referencia son dictados en las regulaciones de agua nacionales o reglamentos locales. Los derechos formalizados en marcos sociolegales locales, por ejemplo, en los (localmente específicos) Reglamentos Internos de Riego, aquí se definen como derechos de referencia locales. Éstos pueden adoptar elementos de derechos de referencia legales.

Los derechos activados (o 'derechos en acción') se refieren a procesos de transformación de los derechos de referencia en reglas y proce-

31 Sección basada en Boelens y Zwarteveen: "Prices and politics...". 
dimientos operativos para la distribución del agua. Los padrones de esa distribución son un resultado de este proceso. Otros productos son las decisiones acerca de quién debe en la práctica tener posibilidad de participar y votar en las organizaciones de usuarios de agua. Los derechos activados son a menudo altamente dinámicos y están sujetos a continuos procesos de regateo, lucha social y disputa. El proceso de activación de derechos no muestra solamente el contenido de los derechos y obligaciones, sino también las variadas maneras en que éstos son interpretados. Por ejemplo, la interpretación generosa y flexible de las sanciones tiende a ser una característica de la mayoría de los sistemas locales andinos, y 'debatir hasta consensuar', más que una aplicación estricta de los derechos de referencia, es una regla general cuando se trata de disputas internas sobre derechos. La activación de los derechos se refiere a las acciones de los usuarios para sobreponerse a los obstáculos que pudieran encontrar en cada una de las esferas de los derechos de agua. Por ejemplo, las mujeres podrían reclamar la construcción de reservorios nocturnos para poder realmente hacer uso de sus derechos de referencia. Para ellas, regar de noche a menudo pone restricciones a la activación de sus derechos.

Los derechos materializados se refieren a las prácticas de uso y distribución del agua y a los procesos reales de toma de decisiones acerca de estas prácticas. Se refieren a las reglas y arreglos como operación entre los usuarios que emergen cuando se usa un sistema de riego. Los derechos materializados con frecuencia no se encuentran escritos o ni siquiera se hacen explícitos. Normalmente son autorizados por la rutina y por acuerdos tácitos o informales.

Tanto la definición de los contenidos de cada uno de estos derechos como los vínculos de transformación de un derecho a otro están sujetos a estructuras y juegos de poder. Los procesos a través de los cuales se materializan los derechos, los escenarios donde esto ocurre y los actores involucrados pueden ser diferentes para las tres categorías de derechos. El proceso desde los derechos de referencia hacia los derechos materializados es también de inclusión y exclusión, no sólo en términos de quién accede al agua, sino también de quién controla o participa en la toma de decisiones. Es al mismo tiempo un proceso técnico, sociolegal y político, que con frecuencia está fuertemente influenciado por argumentos de control culturales o hasta metafísicos. 


\section{La creación y recreación de los derechos de agua}

Antes y después de la construcción del sistema, y durante su uso, los colectivos de usuarios del agua desarrollan su marco local de derechos, un conjunto de normas que guía tanto la creación, la administración y el mantenimiento del sistema como la asignación y la utilización del agua, así como las relaciones entre los usuarios. Por tanto, como se delineó en las secciones anteriores, el mecanismo de inversión de los usuarios para adquirir derechos es con frecuencia extremadamente importante para las comunidades andinas que gestionan sus propios sistemas.

El desarrollo de la infraestructura de riego establece simultáneamente relaciones de propiedad entre los creadores del sistema. Al invertir en las instalaciones, los usuarios crean su 'propiedad hidráulica', una propiedad común del sistema que es el factor que une a los regantes y mueve su acción colectiva. Esto estructura el cimiento que garantiza la realización de las variadas actividades de operación y mantenimiento requeridas en un sistema de riego gestionado por los usuarios. El mecanismo también garantiza a las comunidades indígenas y campesinas, como cuerpos colectivos, que tendrán control efectivo sobre el desarrollo y la aplicación de sus propias normas para gestionar el sistema. ${ }^{32}$ En palabras de Coward: "Las inversiones para crear obras de riego siempre crean o recomponen las relaciones de propiedad con respecto a esas nuevas infraestructuras. En otras palabras, uno no puede crear obras sin establecer propiedad [...] La creación de obras de riego establece relaciones de propiedad entre los creadores, las cuales se vuelven la base social para la acción colectiva en la ejecución de las varias tareas de riego". ${ }^{33}$ Así también, la creación y recreación de derechos de agua en las comunidades andinas se enlaza fuertemente con la formación de identidad.

De este modo, la apropiación de los derechos individuales de las familias es directamente coherente con la apropiación de los derechos colectivos del grupo, y ambos están directamente conectados entre si y son el fundamento básico de la gestión colectiva del sistema. En muchos sistemas

32 También la inversión estatal en el desarrollo de las instalaciones de riego puede verse en el proceso de creación y reforzamiento de propiedad, y las comunidades usuarias lo consideran como tal ya que, tras su inversión, el Estado normalmente fortalece su dominio sobre el recurso hídrico proclamando la materialización de sus derechos de propiedad total y su control sobre el sistema en general.

33 E. Walter Coward Jr.: Property in action. Alternatives for irrigation investment, Khon Kaen, University of Khon Kaen, Thailand, 1983, p.12. 
tradicionales, las familias logran sus derechos de riego no sólo a través de sus propias inversiones contemporáneas en la construcción de las instalaciones colectivas, sino también por herencia de la inversión hecha por sus ancestros y como un acuerdo establecido con las deidades. Esto se confirma a través de los ritos enraizados en la práctica del riego.

Tras generar los derechos, los usuarios deben mantenerlos o conservarlos. Esto lo hacen a través del cumplimiento de sus obligaciones en el sistema de riego, lo que nuevamente toma la forma de una inversión del usuario. La participación en el trabajo colectivo, el pago de deudas, la participación en reuniones, etc. son obligaciones importantes, tanto para conservar los derechos como para hacer que el propio sistema de riego siga funcionando. Sin estas contribuciones obligatorias por parte de cada familia hacia la colectividad, el sistema de riego no es sustentable; la conservación de los derechos tiene un papel clave en la gestión efectiva del sistema de riego. ${ }^{34}$ Así, la inversión de los usuarios en la construcción (o rehabilitación) del sistema está basada en la lógica de que así se crean derechos de agua individuales y colectivos, mientras que la inversión en el mantenimiento reafirma y recrea estos derechos. Las relaciones precisas en términos cuantitativos y cualitativos entre las contribuciones de inversión, la creación y recreación de derechos y la percepción de si esto es equitativo, difieren de sistema a sistema. Esto es un tema esencial en la toma de decisiones a escala local, materia de lucha y negociación intensas, tanto hacia adentro como con terceros.

A pesar de la obvia influencia de la modificación de la infraestructura - y del incremento del caudal - sobre los derechos de agua existentes, muchos proyectos de desarrollo del riego no consideran este tema como algo importante ni lo afrontan explícitamente. Mucho peor entienden la relación entre las inversiones anteriores y las actuales, y la creación de la propiedad hidráulica colectiva. Esta falta de entendimiento y este rechazo de los fundamentos de los sistemas locales a menudo causan la siguiente situación: las contribuciones locales son convocadas, pero sin expresar que estos insumos son inversiones individuales en un sistema de propiedad común, lo que por definición requiere de una clarificación previa acerca de las relaciones entre las contribuciones y los beneficios para cada usuario,

34 El hecho de que la racionalidad de la 'inversión de los usuarios' sea fundamental para sistemas sostenibles no es razón para invocar enfoques románticos. En otras publicaciones hemos analizado las consecuencias para las regantes mujeres que ya están sobrecargadas de trabajo en comunidades donde hay mucha emigración masculina. 
así como un entendimiento compartido acerca de la propiedad en el sistema y los derechos de agua colectivos. Cuando esta inversión desorganizada se acaba, no quedan fundamentos sólidos para que los usuarios organicen la distribución del agua, mucho menos para que mantengan sus canales como propiedad colectiva. ${ }^{35}$

La amalgama y confusión en las relaciones de propiedad existentes son la médula de muchas de las 'fallas de intervención'. Ésa es la razón de que, cuando uno visita un sistema de riego 'posproyecto', no es raro encontrar a los grupos de usuarios todavía discutiendo agriamente sobre las condiciones para acceder al agua y las correspondientes obligaciones de mantenimiento. En tales casos, necesitan inventariar los insumos y establecer las obligaciones requeridas para determinar los derechos de cada usuario a cierta porción del agua. Particularmente problemáticos son los muchos casos en los cuales las agencias externas intervienen e invierten en las obras de riego existentes, fundadas sobre acuerdos de derechos locales y con base en la inversión previa de los usuarios. Estas nuevas inversiones lideradas por agencias, a menudo destruyen los derechos de propiedad colectiva e individual y, consecuentemente, la acción colectiva necesaria para sostener el sistema. El más problemático caso es aquel en que una secuencia de intervenciones implementadas por diferentes instituciones, y manejada con diferentes conceptos de inversión (trabajo comunitario; alimentos por trabajo; mano de obra individual; contribuciones financieras o materiales por derechos de agua, etc.) ha creado un caos institucional entre usuarios antiguos, nuevos y potenciales. El hecho de que muchas contribuciones estén pobremente registradas, sumado a ciertos intereses manipuladores de la información, complica aún más las posibilidades de aclarar la situación. ${ }^{36}$

A diferencia de las nociones comunes de los programas de desarrollo de riego, ya sea paternalistas y verticales o participativos y progresistas, que presentan la participación y las contribuciones locales como un mecanismo para crear un sentimiento de propiedad en los futuros beneficiarios y para garantizar una mejor operación del sistema, los usuarios de los sistemas no pregonan un 'sentimiento de propiedad' sino relaciones reales de propiedad y copropiedad que establecen derechos de acceso y control precisos. Esto también muestra la naturaleza socio-cultural y socio-técnica de los derechos de agua. Los derechos y las relaciones de propiedad están

35 Beccar et al.: "Water rights and collective action...".

36 Ibidem. 
insertados en los territorios culturales, agroecológicos y políticos, donde la creación de derechos colectivos/individuales se entrelaza con la (re)creación de infraestructura y organización, y vitaliza las relaciones de pertenencia mutua. Además muestra la naturaleza política de estos derechos: son objeto de lucha y producto de relaciones de poder. Tanto sus contenidos como su distribución en la sociedad, así como la manera en que se adaptan dinámicamente a las nuevas situaciones, reflejan tanto las estructuras de poder prevalecientes como la manera de afrontarlas.

Crear, consolidar y transformar los derechos de agua: todo es parte del mencionado juego dinámico de poderes. Básicamente, los derechos de agua son una relación de poder entre sujetos, más que sólo una relación entre el sujeto 'usuario' y el objeto 'agua'. En esta relación, algunos interesados obtienen o tienen el derecho (poder autorizado) para actuar de tal manera que influya en los derechos de los otros. Obviamente, en estas relaciones sociales locales de acceso al agua, bajo ciertas condiciones y de acuerdo con los volúmenes establecidos, los flujos y/o los horarios, son un tema nuclear. Pero el gran interés de los varios grupos sociales en controlar el modo en que se definen y asignan los derechos de agua no puede ser explicado solamente a través de la necesidad percibida de acceso al agua y a la infraestructura que la transporta. No, hay más elementos en juego: tener derechos de agua en sistemas de propiedad común también confiere frecuentemente derechos de participar en la toma de decisiones acerca de las reglas de gestión del sistema y de la definición local de los contenidos precisos de los derechos de agua - un segundo nivel de interés en el cual hay un duro enfrentamiento. Y a un tercer nivel vemos una disputa acerca de la legitimidad de los sistemas normativos y sus autoridades: ¿quién tiene el poder legítimo de poner las normas y autorizar las reclamaciones en los sistemas de riego locales? A un cuarto nivel, más abstracto pero no menos importante, están los discursos que defienden regímenes de derechos de agua particulares. Como una conjunción de poder y conocimiento, establecen las aseveraciones sobre 'la verdad' y pretenden crear un alineamiento entre las esferas técnica, organizativa, sociolegal, económico-política y cultural-metafísica del control del agua: para legitimar, afirmar y promover políticas de agua, instituciones de control hídrico y prácticas de distribución de agua particulares. 